\title{
Learning curve analysis of mitral valve repair using telemanipulative technology
}

\author{
Patrick J. Charland, MD, MBA, ${ }^{\mathrm{a}}$ Tom Robbins, PhD, ${ }^{\mathrm{b}}$ Evilio Rodriguez, MD, FACS,,${ }^{\mathrm{ac}}$ \\ Wiley L. Nifong, MD, FACS, ${ }^{\mathrm{a}}$ and Randolph W. Chitwood, Jr, MD, FACS ${ }^{\mathrm{a}, \mathrm{c}}$
}

Objective: To determine if the time required to perform mitral valve repairs using telemanipulation technology decreases with experience and how that decrease is influenced by patient and procedure variables.

\begin{abstract}
Methods: A single-center retrospective review was conducted using perioperative and outcomes data collected contemporaneously on 458 mitral valve repair surgeries using telemanipulative technology. A regression model was constructed to assess learning with this technology and predict total robot time using multiple predictive variables. Statistical analysis was used to determine if models were significantly useful, to rule out correlation between predictor variables, and to identify terms that did not contribute to the prediction of total robot time.
\end{abstract}

Results: We found a statistically significant learning curve $(P<.01)$. The institutional learning percentage* derived from total robot times $\dagger$ for the first 458 recorded cases of mitral valve repair using telemanipulative technology is $95 \%\left(\mathrm{R}^{2}=.40\right)$. More than one third of the variability in total robot time can be explained through our model using the following variables: type of repair (chordal procedures, ablations, and leaflet resections), band size, use of clips alone in band implantation, and the presence of a fellow at bedside $(P<.01)$.

Conclusions: Learning in mitral valve repair surgery using telemanipulative technology occurs at the East Carolina Heart Institute according to a logarithmic curve, with a learning percentage of $95 \%$. From our regression output, we can make an approximate prediction of total robot time using an additive model. These metrics can be used by programs for benchmarking to manage the implementation of this new technology, as well as for capacity planning, scheduling, and capital budget analysis. (J Thorac Cardiovasc Surg 2011;142:404-10)

Hermann Ebbinghaus ${ }^{1}$ was the first to describe learning effects using empiric methods in his 1885 article Memory: A Contribution to Experimental Psychology. Since then, the learning curve has been studied in many fields, from manufacturing to surgery. ${ }^{2-7}$ Learning curve analysis has been used in manufacturing as a way to forecast costs and project completion dates. Further, it has been used as a mechanism for maintaining labor efficiency through goal setting and benchmarking. It has also helped to prevent delay in bringing problems to the attention of management. ${ }^{4}$ With the initiation of telemanipulation technology in cardiothoracic surgery, data on learning curves could be helpful to surgeons and administrators alike in managing technology implementation, training surgeons, ${ }^{8,9}$ capacity planning, ${ }^{4,6}$ scheduling, ${ }^{4}$ and business forecasting. ${ }^{4,10}$

The use of telemanipulation technology has grown rapidly since its introduction in surgery. ${ }^{11}$ Studies presented in the journal Management Science have looked at learning

From the East Carolina Heart Institute, ${ }^{\text {a }}$ Greenville, NC; The School of Business at East Carolina University, ${ }^{\mathrm{b}}$ Greenville, NC; and University Health Systems of Eastern North Carolina, ${ }^{\mathrm{c}}$ Greenville, NC.

Disclosures: Authors have nothing to disclose with regard to commercial support.

Received for publication July 9, 2010; revisions received Sept 29, 2010; accepted for publication Oct 17, 2010; available ahead of print Dec 20, 2010.

Address for reprints: Patrick J. Charland, MD, MBA, 5516 Solomons Seal Court, Holly Springs, NC 27540 (E-mail: pjc1020@yahoo.com).

$0022-5223 / \$ 36.00$

Copyright $($ c 2011 by The American Association for Thoracic Surgery doi:10.1016/j.jtcvs.2010.10.029 associated with this technology in cardiothoracic surgery and shown that the learning curves can differ significantly from one institution to another. ${ }^{5}$ These studies have not incorporated specific aspects of learning related to the type of mitral valve repair procedure performed. At the East Carolina Heart Institute, more than 500 heart operations have been performed using the da Vinci Surgical System (Intuitive Surgical Inc, Sunnyvale, Calif), and our prospective database has provided a unique opportunity to perform a statistical evaluation of high-volume institutional learning during complex valve repairs.

\section{MATERIALS AND METHODS}

Between May 2000 and September 2009, 500 patients underwent mitral valve repair surgery using minimally invasive telemanipulative technology (da Vinci Surgical System). A single-center retrospective review was conducted using preoperative, intraoperative, and postoperative data that were collected prospectively according to approved institutional review board protocol and archived in the Robotic Mitral Valve Repair or Society of Thoracic Surgeons' database.

The objective of our analysis was to determine if the time required to perform mitral valve repairs using telemanipulation technology decreases with experience and how that decrease is influenced by patient and

\footnotetext{
* Learning percentage: the percentage decrease in operative time each time case number doubles. Example: If the learning percentage is $50 \%$ and case 1 takes 100 minutes to perform, case 2 will take 50 minutes, case 4 will take 25 minutes, case 8 will take 12.5 minutes, case 16 will take 6.25 minutes, and so on until the asymptote is reached.

$\dagger$ Total robot time: starts when the da Vinci Surgical System connects to the patient and ends when the da Vinci Surgical System disconnects from the patient.
} 


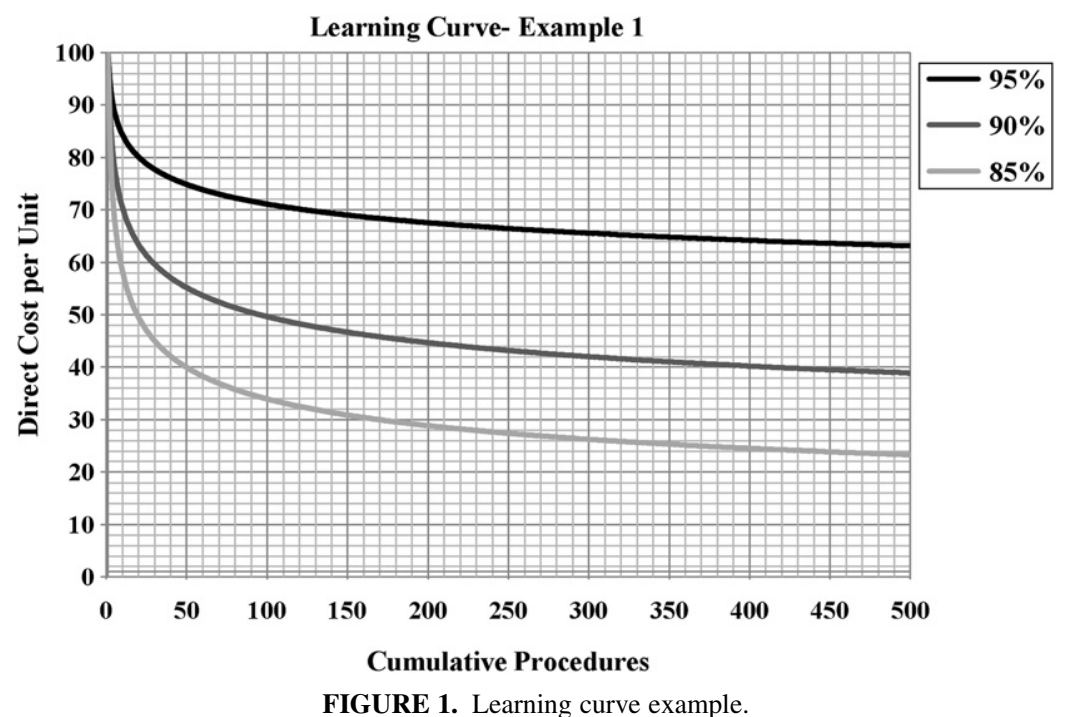

procedure variables. We hypothesize that learning occurs according to a logarithmic curve of the form,

$$
Y_{x}=K x^{n},
$$

where $\mathrm{Y}$ represents the total robot time required to perform a procedure, $x$ represents the sequential procedure number, $K$ represents the time required for the first procedure, and $n$ represents a learning rate. More precisely, $n$ is calculated as

$$
n=\log b / \log 2
$$

where $b$ is the learning percentage. With each doubling of case number, there is a decrease in the total robot time to some percentage of the time from the case number being doubled; this percentage is the learning percentage. This functional form creates a familiar learning curve, where procedure times decrease with cumulative output at a decreasing rate as illustrated in Figure 1. Estimating the learning curve is facilitated by taking the logarithm of the procedure number and the procedure time. This transforms the nonlinear curve shown in Figure 1 into the linear curve shown in Figure 2.

To determine our institutional learning percentage, we performed a linear regression analysis using the log of total robot time as the dependent variable and the $\log$ of the procedure number as the independent variable. The resulting model was not statistically significant, with an overall $P$ value greater than $33 \%$ and an $\mathrm{R}^{2}$ value less than $1 \% .^{12,13}$

We next considered the possibility that other factors may be affecting the overall procedure time and obscuring any learning effect. By using archived data, we collected 19 variables about the patient and the procedures performed. Cases were differentiated on the basis of a classification of 5 different procedures, including band (A), chordal (B), atrial fibrillation ablation (C), leaflet resection (D), and tissue approximation (E) (complete description in "Terminology" section). The procedure type, annuloplasty band size, band type, da Vinci model, presence of a training fellow, presence of 2 surgeries in the same day, and use of clips alone in band implantation were considered, as were patient characteristics such as body mass index, age, and gender.

Statistical tests were used to determine if models were statistically meaningful (global F-test) to test for correlation between independent variables (variance inflation factors), to exclude terms that did not contribute to the prediction of total robot time ( $t$ tests), and to determine if the residuals can be assumed to follow a normal distribution. The resulting regression model (Table 1) contains the following 7 significant independent covariates:
- Log total robot time: the $\log$ (base 10) of total robot time in hours. Total robot time is the amount of time the robot was used in the procedure. It begins when the robot connects to the patient and ends when the robot disconnects from the patient. Total robot time varied from 0.38 hours to 3.48 hours with an average of 1.49. Log (total robot time) varies from -0.424 to 0.542 with an average of 0.146 .

- Fellow: represents the presence of a fellow at bedside. There were 15 different fellows present over the course of the procedures performed. Data were coded such that 1 indicates presence of a training fellow assisting at the bedside and 0 indicates no fellow present. A fellow was present in $35 \%$ of the procedures.

- Clips: represents cases using nitinol U-clips (Coalescent Surgical, Sunnyvale, Calif) exclusively in annuloplasty band implantation. This occurred in 114 cases, whereas sutures alone were used 261 cases and both were used in 84 cases. Prior studies have shown a significant reduction in annuloplasty band implantation time* with the use of nitinol clips. ${ }^{14,15}$

- Band size: represents the size of the annuloplasty band used in the mitral valve repair. For each case, the size of the band in millimeters was listed in the regression. Band sizes ranged from 25 to $40 \mathrm{~mm}$. Our surgeon's average band implantation rate was $1.04 \pm 0.31$ $\mathrm{mm} / \mathrm{min}(\mathrm{n}=460)$. When band size (millimeters) was graphed against the number of clips and sutures used per band, there was a positive relationship $(\mathrm{y}=1.02 \times+21.5)$.

- Chordal: represents whether or not the mitral valve repair for that case included a chordal (type B) procedure in addition to the band implantation. If so, 1 unit was assigned for each type B procedure performed during the case, and if not, 0 was assigned. Type $B$ procedures were performed in $42 \%$ of the cases.

- Ablation: represents whether or not the mitral valve repair for that case included an atrial fibrillation ablation (type C) procedure in addition to band implantation. If so, 1 was assigned, and if not, 0 was assigned. This procedure type was performed in approximately $19 \%$ of the cases.

- Resection: represents whether or not the mitral valve repair for that case included a leaflet resection (type D) procedure in addition to band implantation. If so, 1 unit was assigned for each type $\mathrm{D}$ procedure performed during the case, and if not, 0 was assigned. Type D procedures were performed in $68 \%$ of the cases.

\footnotetext{
* Band implantation time: starts when band is in position and ends after last suture is placed in annuloplasty band.
} 


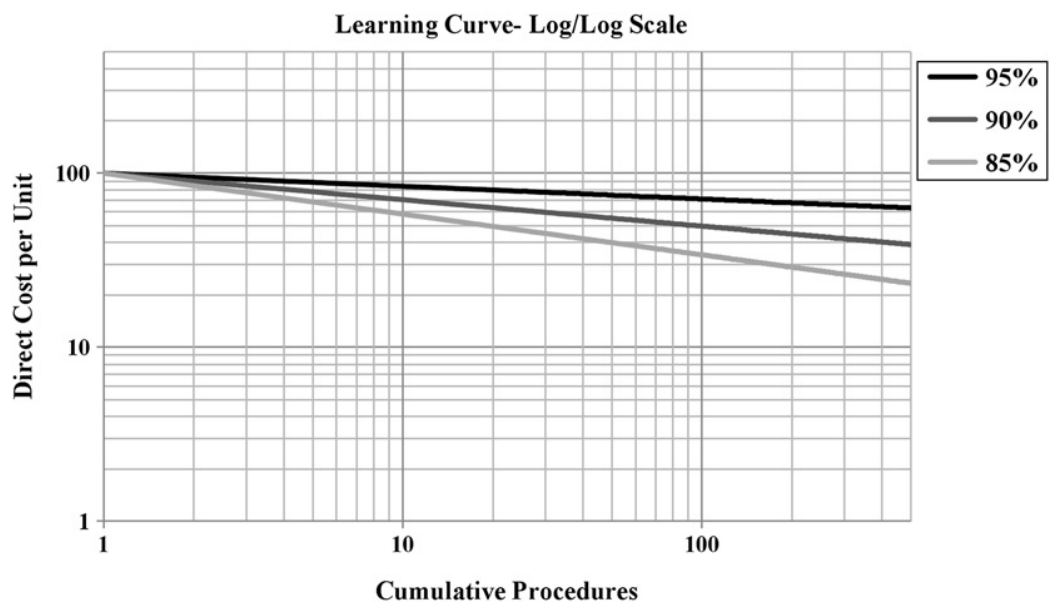

FIGURE 2. Learning curve example $\log / \log$ scale.

Our institutional learning percentage was calculated using the coefficient associated with the $\log$ of procedure number $(\log \mathrm{n})$ displayed in the regression output from our final model (Table 1) to solve for $b$ in equation (1.1) ${ }^{16}$ Incremental times (Table 2) were then determined using the coefficients from the other significant independent variables in our regression output.

The effect of learning on total robot time was further studied by considering the effect of procedure complexity. To examine this, a value for procedure complexity (Figure 3) was created by summing the number of procedures of each type (B-E) performed in the case, multiplied by its incremental time. These values were then plotted to show the positive correlation with case number.
Next, an additive model was used to create a predictive equation (2.0) to estimate total robot time based on case number and influential variables. Residuals and a standard deviation were calculated from predicted total robot times and total robot times, and these data were found to follow a normal distribution. With this information, a $95 \%$ confidence interval was determined for total robot times predicted by the equation.

\section{RESULTS}

By using Excel software (Microsoft Corp, Redmond, Wash) with statistical analysis add-in to perform regression

TABLE 1. Regression analysis printout from Excel (Microsoft Corp, Redmond, Wash)

Regression statistics

\begin{tabular}{lllc}
$\mathrm{R}^{2}$ & 0.398 & & \\
Adjusted $\mathrm{R}^{2}$ & 0.389 & $\mathrm{n}$ & 458 \\
$\mathrm{R}$ & 0.631 & $\mathrm{k}$ & 7 \\
$\mathrm{SD}$ & 0.124 & Dep. Var. & $\log \operatorname{trt}$ \\
\hline
\end{tabular}

ANOVA table

\begin{tabular}{lrrrrr}
\hline \multicolumn{1}{c}{ Source } & SS & df & MS & F & P value \\
\hline Regression & 4.5688 & 7 & 0.6527 & 42.54 & $5.62 \mathrm{E}-46$ \\
Residual & 6.9045 & 450 & 0.0153 & & \\
Total & 11.4733 & 457 & & & \\
\hline
\end{tabular}

Regression output

Confidence interval

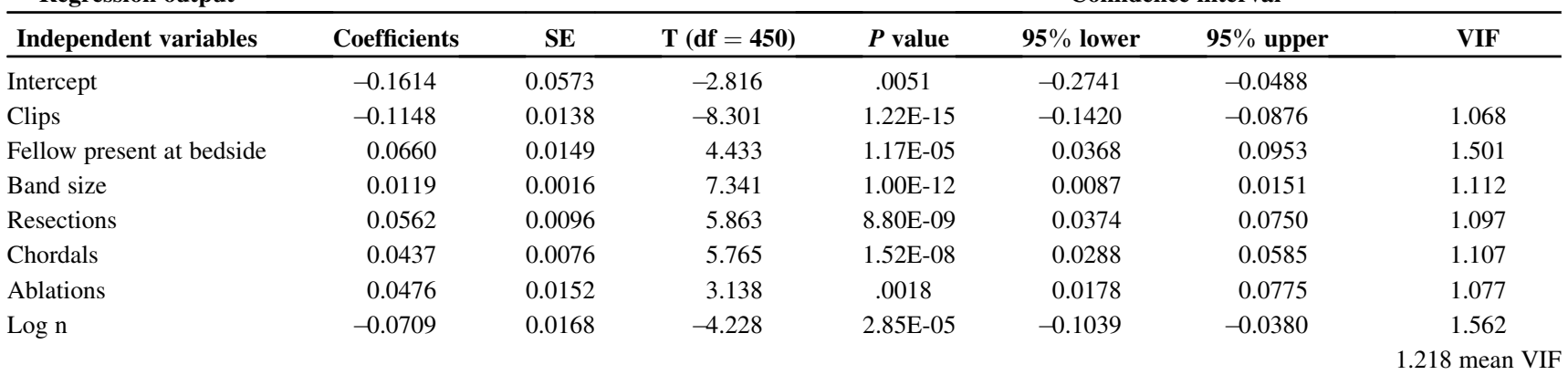

ANOVA, Analysis of variance; $S E$, standard of error; $S D$, standard deviation; $M S$, mean squared; $S S$, sum of squares; $d f$, degrees of freedom; $k$, number of independent variables; $F$, f ratio; VIF, variance inflation factor; Dep. Var. $\log t r t$, the dependent variable is the log of total robot time. 
TABLE 2. Coefficients and incremental times for independent variables

\begin{tabular}{lcc}
\hline Independent variable & Coefficient & $\begin{array}{c}\text { Incremental } \\
\text { time (h) }\end{array}$ \\
\hline Resections & 0.0562 & 1.138133 \\
Atrial fibrillation ablations & 0.0476 & 1.115937 \\
Chordals & 0.0437 & 1.105749 \\
Clips & -0.1148 & 0.767688 \\
Fellow at bedside & 0.0660 & 1.1642 \\
Band size & 0.0119 & 1.027713 \\
\hline
\end{tabular}

analysis, we reached a statistically significant model (Table 1) containing 7 independent variables with a learning percentage of $95 \%(P<.01)$. As shown in Figure 1, a learning percentage between $85 \%$ and $95 \%$ will result in a substantial decrease in procedure time over the course of 500 procedures.

Our model explains more than one third of the variability in total robot time $\left(\mathrm{R}^{2}=0.40\right)$. The model assumes that times for individual procedures follow a normal distribution with a standard deviation of $0.125 \log$ hours. Statistical theory tells us that $95 \%$ of normally distributed outcomes fall within 2 standard deviations of the mean; therefore, our model can be used to make approximate predictions in total robot time using the following equation $(2.0)^{13}$ :

$$
\begin{aligned}
\log (\text { TRT }) & =(0.0660 * \text { Fellow })+(0.0119 * \text { Band Size }) \\
+ & (0.0437 * \text { Type B })+(0.0476 * \text { Type C }) \\
+ & (0.0562 * \text { Type } \mathrm{D})+(-0.1148 * \text { Clip }) \\
& +(-0.0710 * \log (\mathrm{n}))+(-0.1614)
\end{aligned}
$$

The 7 significant variables in our model include the presence of a fellow, annuloplasty band size, $\log \mathrm{n}$, use of clips alone in band implantation, inclusion of chordal procedure (B), atrial fibrillation ablations (C), and leaflet resections (D) $(P<.01)$. Variance inflation factors in the regression output (Table 1) are all less than 2 , with the average being 1.28 (variance inflation factors $>10$, and in some textbooks 5 are considered problematic), indicating there are no significant problems with multicollinearity in our model. ${ }^{13,16}$

Patient age, sex, and body mass index were not found to be significant predictors in initial trial models $(P>.05)$. Procedure factors such as da Vinci model, 2 cases in 1 day, a tissue approximation procedure, and band type were not significant. $P$ values determined for the nonsignificant variables are listed in Table 3.

Our surgeon's experience has shown that procedure complexity increases with case number $(y=0.003 \times+1.417)$. This is illustrated in Figure 3.

The number of robotic cases performed by the primary surgeon in this study has increased relative to the number of non-robotic cases over the last decade. In 2000, the surgeon performed 14 robotic mitral repairs compared with 52 non-robotic mitral valve repairs, whereas in 2009 there were 90 robotic mitral valve repairs performed and 14 nonrobotic mitral valve repairs.

\section{DISCUSSION \\ Organizational Learning}

Literature on this topic states that different programs may progress at different rates depending on whether they capitalize on their experience. Pisano and colleagues ${ }^{5}$ Management

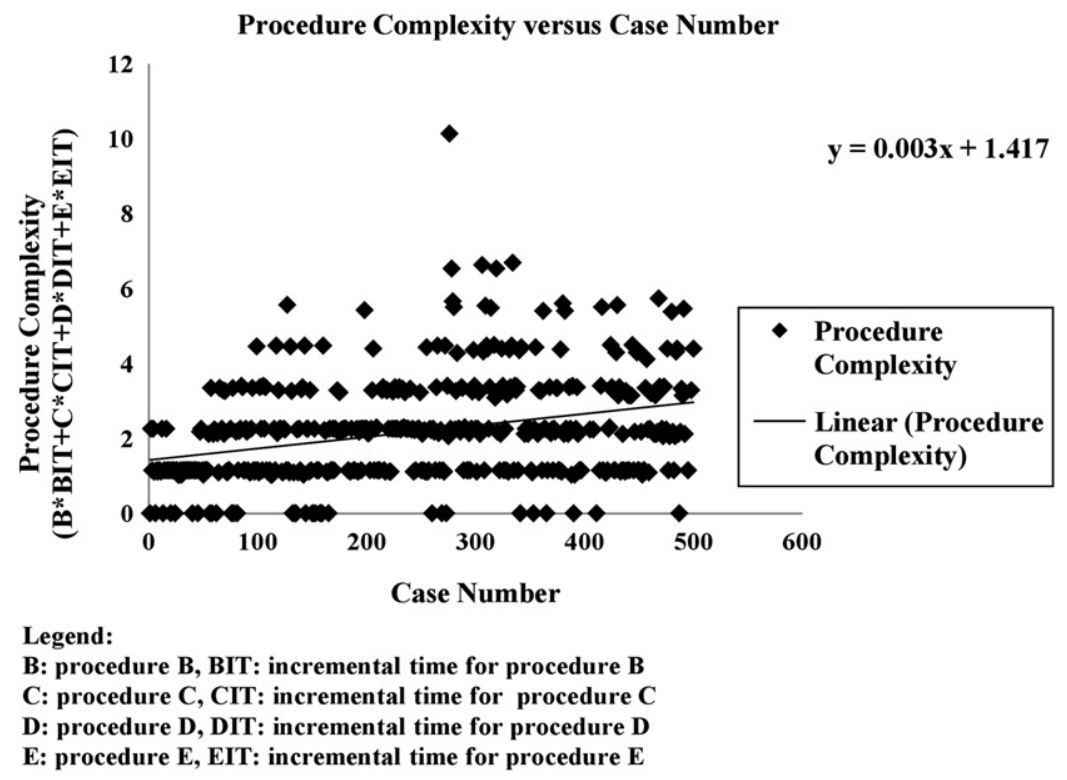

FIGURE 3. Procedure complexity versus case number. 
TABLE 3. Results of $t$ test for nonsignificant variables

\begin{tabular}{lc}
\hline \multicolumn{1}{c}{ Independent variable tested not significant } & $\boldsymbol{P}$ value \\
\hline Age & .20 \\
BMI & .08 \\
Gender & .08 \\
da Vinci model (Intuitive Surgical Inc, Sunnyvale, Calif) & .08 \\
Tissue approximations & .30 \\
Cosgrove-Edwards Classic Annuloplasty Ring & .09 \\
$\quad$ (Edwards Lifesciences LLC, Irvine, Calif) & \\
Carpentier-Edwards Physio Annuloplasty System Ring & .74 \\
$\quad$ (Edwards Lifesciences LLC) & .74 \\
UNIRING, Universal Annuloplasty System & \\
$\quad$ (Peters Surgical, Bobigny Cedex, France) & .73 \\
ATS Simulus Flex-C Band & \\
$\quad$ (ATS Medical Inc, Minneapolis, Minn) & .67 \\
ATS Simulus Semi-Rigid Annuloplasty Ring & .08 \\
$\quad$ (ATS Medical Inc) & \\
ATS Simulus Flexible Annuloplasty Ring & .44 \\
$\quad$ (ATS Medical Inc) & \\
Performing 2 surgeries in 1 d & \\
Robotic atriotomy & .72 \\
\hline BMI Body mass index. & \\
\hline
\end{tabular}

Science study, Organizational Differences in Rates of Learning, suggests that "certain differences may be rooted in structural and organizational aspects of the adopters themselves and may not be subject to change by the innovator," and further that "unless an organization puts into place mechanisms for capturing knowledge and implementing learning, experience may not translate into competence."

\section{Capitalizing on Experience}

Our large case volume allows us a unique opportunity to examine our experience and obtain sound observations that should contribute to better technology implementation. ${ }^{17}$ Our learning percentage indicates that there is learning at our institution with this technology. To understand the learning process and capitalize on our experience, we have tried to further examine the factors involved with learning that affect total robot time. Specifically, we have classified the types of procedures performed in each case and determined an incremental time to perform them. We have done this to obtain insight, which will assist us in harnessing the best qualities of this technology to achieve the best possible patient care.

\section{Effect of Procedure Type on Total Robot Time}

Each independent variable contributes a unique incremental time to the base band implantation procedure (Table 2). Incremental times for grouped procedure types indicate that leaflet resections (D) are the most timeconsuming, followed by atrial fibrillation ablations (epicardial alone, endocardial alone, and epicardial combined with endocardial ablations were coded the same) (C) and chordal procedures (B). This is not a direct measure of technical difficulty. Incremental times are derived from total robot times of cases coded to have included different procedures over 500 cases. They are meant to offer an understanding of how total robot time is affected by procedure type, and to allow for its prediction. These procedure groupings do not vary by large time intervals. However, when compared with tissue approximations (E), the incremental times are significant.

It may be necessary to perform multiple procedure types during a mitral valve repair case to achieve the best repair for the patient, whereas other cases will require less. This variation has a notable effect on total robot times. From the procedure complexity graph (Figure 3), one can see that as case volume increases, the surgeon tends to perform more complex repairs. This helps to explain why the plot of actual total robot time versus case number (Figure 4) does not show a dramatic and consistent decrease in total robot time when we know that learning is occurring.

The effect of other independent variables on total robot time also will vary from case to case. For example, there may or may not be a fellow present, band size may be large or small depending on the needs of the patient, and sutures may be required in band implantation. This also explains much of the variation in total robot time from case to case and the lack of a consistent decrease in total robot time with increasing case number. Although the presence of a fellow at bedside adds significant time to the procedure, the education value to the trainee and its impact on future patients is certainly worthwhile.

\section{Predictive Utility of the Model}

Given that significant variables fluctuate widely from case to case depending on patient needs, we have developed an equation for predicting total robot time that takes this into account.

The graph of predicted total robot times versus actual total robot times in Figure 5 illustrates the tight fit between the

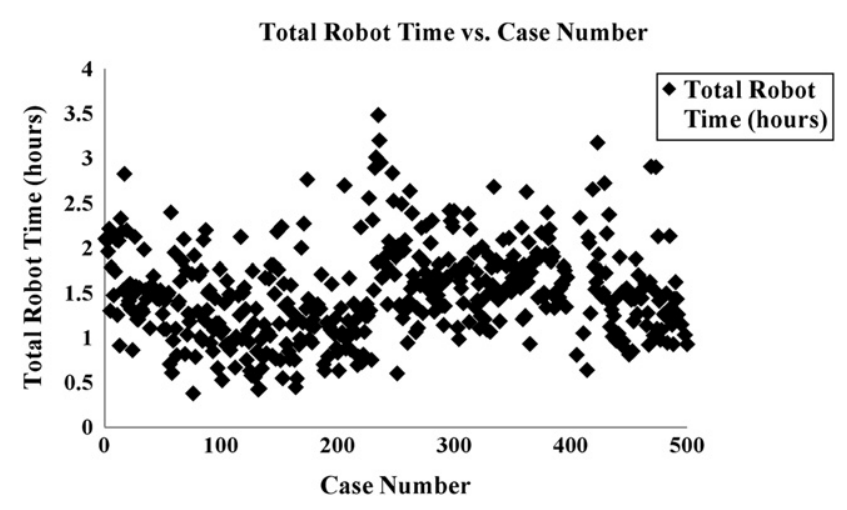

FIGURE 4. Total robot time versus case number. 
values derived from the predictive equation (2.0) and our actual results.

The predictive equation can be used in many ways because it allows for other programs to benchmark our experience. It can be used by the primary surgeon to take control of his/her team's progress; to set goals; to guide training; to improve efficiency, team work, coordination, and organization; and to provide reassurance or motivation.

\section{Applying the Equation}

Being accurately aware of how operative times change with different variables can help surgeons beginning with the technology to approach cases with greater understanding of the procedure time requirements. By having fairly similar incremental times, these procedure types could be interchanged, but the combination of significant procedures to be performed by the trainee would ideally progress in a stepwise fashion, as is feasibly possible through patient selection and faculty mentor coordination. ${ }^{18,19}$ For example, the data suggest it would be best for a surgeon new to the technology to choose cases first requiring tissue approximation or annuloplasty band alone. Next, it would be logical to accept cases with annuloplasty along with a chordal procedure or ablation. As a surgeon progresses, and operative times are equal or less than that predicted by our equation, the trainee can comfortably add complexity, such as a case with a band, chordal, and atrial fibrillation ablation \pm tissue approximation. When operative times are not being met, the surgeon would seek to define what is impairing the team from meeting its goal, and guide them to make the necessary changes.

Operative times are merely one variable to consider in training and technology implementation, however. These data are not meant to supplant the oversight of experienced surgeon mentors who can assess other variables involved with measuring competency, such as with decisionmaking skills when complications arise.

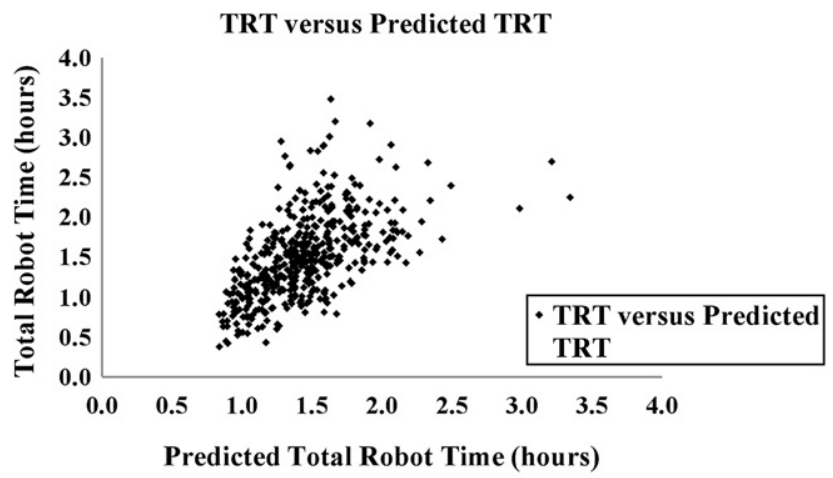

TRT: total robot time

FIGURE 5. Predicted total robot time versus actual total robot time. TRT, Total robot time.

\section{Applications in Surgical Training}

There has been recent interest in surgical training methods and evaluation techniques because surgeons in this field must often adopt new technology to supplement and advance their skills. ${ }^{5}$ With the recent incorporation of the Joint Council on Thoracic Surgery Education and its current initiative to develop and initiate innovative teaching methods, many solutions have been offered. ${ }^{5}$ For example, a high-fidelity cardiac surgical simulator is being used by integrated thoracic surgery residents at the initiation "boot camp" of their residency each year. ${ }^{6,7}$ Various simulator models are being used to train surgeons for beating heart coronary artery surgery and other procedures. ${ }^{7-9}$

Recent literature has suggested the use of simulators in competency-based testing and certification. ${ }^{10}$ Performance on simulators has been evaluated with a comparison of times from initial experience to later experience or through the use of a 3-point psychometric grading scale. ${ }^{6,9,11}$

Multiple regression analysis was used in this study to map out the learning curve for mitral valve repair surgery using telemanipulative technology. This technique could also be applied to other procedures using simulators to mimic the operative environment, providing a metric to evaluate progress and competency of trainees, and a means to evaluate factors affecting their progress. Further, data from this type of analysis could be used in the certification process with competency-based testing.

\section{Data Quality}

We encountered minor database quality issues in our process. Data that were deemed unacceptable to a high standard in quality was left out of our analysis. Of the total 500 cases performed to completion using the da Vinci model, we had reliable data on only 458 of the cases, because 7 cases were partially recorded, 4 cases did not use the da Vinci model exclusively for the entire case, and 31 cases were not recorded. Records show that approximately 30 cases used the da Vinci model for atriotomy, whereas presumably the rest were done by hand; however, its use in these cases did not have a significant impact on total robot time $(P=.08)$. The conclusions of our analysis are believed to reflect our true experience.

\section{Study Limitations}

Conclusions should be tempered by the fact that they are based on analysis of a single institution's experience. However, our sample size is substantial for the application of this technology in cardiac surgery, and our results allow for a valuable comparison.

\section{CONCLUSIONS}

Learning mitral valve surgery using telemanipulative technology proceeds according to a logarithmic curve, 
with a learning percentage of $95 \%$. Multiple regression analysis allowed us to define 7 significant variables that affect total robot time and offered us an awareness of how procedure complexity can affect total robot time.

Predictions of total robot time can be approximately estimated with our equation, which can be used as a metric whereby surgeons can manage and evaluate their team's progress in the implementation of this technology. ${ }^{4}$ In addition, hospital administrators may consider its use for capacity planning, scheduling, and capital budgeting analysis. ${ }^{10}$

\section{References}

1. Ebbinghaus H. Influence of repeated learning. In: Memory: A Contribution to Experimental Psychology. New York: Dover Publications; 1964:82-9.

2. Argote L, Epple D. Learning curves in manufacturing. Science. 1990;247:920-4.

3. Cherrington JE, Lippert S, Towill DR. The effect of prior experience on learning curve parameters. Int J Prod Res. 1987;25:399-411.

4. Garvin DA, Field LC, Simpson J. Boeing 767: From Concept to Production (A). Boston: Harvard Business School Publishing; 1991

5. Pisano GP, Bohmer RMJ, Edmondson AC. Organizational differences in rates of learning: evidence from the adoption of minimally invasive cardiac surgery. Manage Sci. 2001;47:752-68.

6. Jacobs RF, Chase RB, Aquilano NJ. Operations \& Supply Chain Management. 12th ed. Boston and New York: McGraw-Hill/Irwin Series Operations and Decision Sciences; 2008.

7. Kaul S, Shah N, Menon M. Learning curve using robotic surgery. Curr Urol Rep. 2006;7:125.

8. Passerotti CC, Passerotti AM, Dall'Oglio MF, Leite KR, Nunes RL, Srougi M, et al. Comparing the quality of the suture anastomosis and the learning curves associated with performing open, freehand, and robotic- assisted laparoscopic pyeloplasty in a swine animal. J Am Coll Surg. 2009; 208:576-86.

9. Carpenter A, Yang C, Uhlig P, Colson Y. Envisioning simulation in the future of thoracic surgical education. J Thorac Cardiovasc Surg. 2008;135: 477-84.

10. Ross SA, Westerfield RW, Jordan BD. Fundamentals of Corporate Finance. 8th ed. Boston; New York: McGraw-Hill/Irwin; 2008.

11. Intuitive Surgical, “2009 Annual Report,” Securities and Exchange Commission Filing. Available at: https://materials.proxyvote.com/Approved/46120E/ 20100222/AR_53320/HTML2/intuitive_surgical-ar2009_0002.htm. Accessed July 26, 2010.

12. Hemphill JF. Interpreting the magnitudes of correlation coefficients. Am Psychol. 2003;58:78-9.

13. McClave JT, Benson PG, Sincich T. Statistics for Business and Economics. 10th ed. Upper Saddle River, NJ: Prentice Hall; 2008.

14. Cook R, Nifong L, Enterkin J, Charland P, Reade C, Kypson A, et al. Significant reduction in Annuloplasty operative time with the use of nitinol u-clips in robotically assisted mitral valve repair. J Thorac Cardiovasc Surg. 2007; 133:1264-7.

15. Reade C, Bower C, Maziarz D, Conquest A, Sun Y, Nifong L, et al. Sutureless robot-assisted mitral valve repair: an animal model. Heart Surg Forum. 2003; 6:254-7.

16. Kutner MH, Nachtsheim C, Neter J, Neter L. Applied Linear Statistical Models. Boston; New York: McGraw-Hill/Irwin; 2007.

17. Chitwood W, Rodriguez E, Chu M, Hassan A, Ferguson T, Vos P, et al. Robotic mitral valve repairs in 300 patients: a single center experience. J Thorac Cardiovasc Surg. 2008;136:436-41.

18. Cook RC, Nifong LW, Lashley GG, Duncan RA, Campbell JA, Law YB, et al. Echocardiographic measurements alone do not provide accurate non-invasive selection of annuloplasty band size for robotic mitral valve repair. J Heart Valve Dis. 2006; 15:524-7.

19. Jones B, Krueger S, Howell D, Meinecke B, Dunn S. Robotic mitral valve repair: a community hospital experience. Tex Heart Inst J. 2005; 32:143-6.

\section{TERMINOLOGY}

Type A procedure: annuloplasty band implantation (using sutures, clips, or a combination).

Type B procedure: chordal procedure (transposition, transplant, replacement, shortening, removal, reattachment, papillary muscle repair).

Type C procedure: ablation for atrial fibrillation (epicardial alone, endocardial alone, and epicardial and endocardial ablations combined) location. Variations in ablation placement were not coded separately. Procedures received 1 if an ablation was performed or 0 if no ablation was performed.

Type D procedure: leaflet resection (quadrangular, triangular, or trapezoidal) with and without repair or sliding plasty. Also in this group: commissurotomy, commissuroplasty, annular decalcification, leaflet perforation repair, leaflet imbrication or plication, leaflet reduction, and leaflet debridement.

Type E procedure: tissue approximation (patent foramen ovale closure, atrial appendage closure, Alfieri edgeto-edge, superior vena caval repair, annular compression, annular plication, cleft closure). 